\title{
(3) Científic@ e-ISSN 2358-26oX \\ AVALIAÇÃO DE FUNGICIDAS NO CONTROLE DE MANCHA ALVO E NO RENDIMENTO DA CULTURA DA SOJA PARA A REGIÃO DE RIO VERDE
}

\author{
EVALUATION OF FUNGICIDES IN TARGET STAIN CONTROL AND SOYBEAN YIELD FOR THE RIO \\ VERDE REGION \\ Rogério Castro Mendonça ${ }^{1 *}$, Luís Henrique Carregal Pereira da Silva ${ }^{1}$; Luiz Cesar Lopes Filho ${ }^{2}$
}

${ }^{1}$ AgroCarregal - Rio Verde

${ }^{2}$ Universidade Federal de Goiás

\section{Info}

Recebido: 05/2021

Publicado: 06/2021

DOI: $10.37951 / 2358-260 X .2021 \mathrm{v} 8 \mathrm{i} 1.5818$

ISSN: 2358-260X

\section{Palavras-Chave}

Fungicidas; Glycine max; Corynespora

cassiicola.

Keywords:

Fungicides; Glycine max; Corynespora casiicola.

\section{Resumo}

A cultura da soja (Glycine max) tem diversificado cada vez mais em relação ao padrão de planta (porte físico). Sendo assim devemos prevenir nas medidas de controle das doenças ocorrentes. Esse trabalho tem ênfase na doença Mancha Alvo, causada pelo fungo Corynespora casiicola, que pode causar perdas significativas na cultura. Utilizou-se a cultivar CD 2728, a qual é suscetivel a esse patógeno, com 6 tratamentos: T1 - Testemunha, T2 - Bixafen + Protioconazol + Trifloxistrobina, T3 - Fluxapiroxade + Protioconazol, T4 - Azoxistrobina + Tebuconazol + Mancozebe, T5 - Azoxistrobina + Protioconazol + Mancozebe e T6 - Protioconazol + Fluindapyr. $O$ controle químico de doenças de plantas é na maioria das situações a medida mais eficiente e viável economicamente para garantir uma alta produtividade, não esquecendo que às condições climáticas para realização do trabalho deve ser favorável para a doença. Desta forma o presente trabalho utilizou os mesmos fungicidas para cada tratamento em todas as aplicações, com objetivo de avaliar a eficácia deles no controle de Mancha Alvo. Outro fator importante que devemos salientar é a necessidade de rotação dos grupos químicos, ingredientes ativos, além da utilização de multissítios dentro dos programas de aplicações.

\section{Abstract}

The soybean crop (Glycine max) has diversified more and more in relation to the plant pattern (physical size). Therefore, we must prevent the control measures of the disease that are current. This work has emphasis on the disease Target Spot, caused by the fungus Corynespora casiicola, which can cause significant losses in the crop. The cultivar CD 2728 was used, which is susceptible to this pathogen, with 6 treatments: T1 - Control, T2 - Bixafen + Protioconazole + Trifloxistrobin, T3 - Fluxapiroxade + Protioconazole, T4 - Azoxistrobina + Tebuconazole + Mancozeb, T5 - Azoxistrobin + Protioconazole + Mancozebe and T6 - Protioconazole + Fluindapyr. The chemical control of plant diseases is in most situations the most efficient and economically viable measure to ensure a high productivity, not forgetting that the climatic conditions to perform the work should be favorable for the disease. Thus, the present study used the same fungicides for each treatment in all applications, in order to evaluate their efficacy in the control of Target Spot. Another important factor that we should highlight is the need for rotation of chemical groups, active ingredients, in addition to the use of multisites within application programs.

\section{INTRODUÇÃO}

Nos últimos anos a cultura da soja passou por profundas transformações, principalmente no que se refere a ciclo, hábito de crescimento, índice de área foliar e potencial produtivo. Tais mudanças acarretaram também na dinâmica das doenças e, consequentemente, na implementação de medidas de controle (Godoy et al., 2020). A produção de alimentos enfrenta diversos desafios, como o uso eficiente de insumos, principalmente em relação ao uso indiscriminado de fungicidas e métodos sustentáveis para adaptação das culturas (Sentelhas et al. 2015; St-Marseille et al., 2019). Doenças tidas como secundárias passaram a ter importância primária dependendo da região de cultivo, condições ambientais e tratos culturais adotados. 
A doença conhecida como Mancha-alvo tem crescido em importância nas diferentes regiões do país, causando perdas significativas na cultura (Flor et al., 2018; Marques et al., 2019). A mancha alvo da soja Glycine max (L.) Merr. é causada pelo fungo Corynespora cassiicola (Berk. \& M.A. Curtis) C.T. Wei (Soares et al., 2009). Trabalhos a campo têm evidenciado os problemas com controle químico desde a safra 2007/08 onde foi confirmado que os benzimidazóis não eram mais efetivos no controle da doença (Godoy, C. V. et al..2007). A situação foi confirmada posteriormente com vários testes de isolados de diferentes regiões do Brasil (Avozani et al., 2014). Também há relatos de resistência ao grupo químico das estrobilurinas e, mais recentemente, foi reportado a resistência às carboxamidas (Terramoto et al., 2017).

É nesse contexto que as recomendações de controle químico também devem ser adaptadas para a nova situação, principalmente em relação aos fungicidas utilizados. O controle químico de doenças das plantas é na maioria das situações a medida mais eficiente $\mathrm{e}$ economicamente viável para garantir produtividade e qualidade da produção (Amorim et al., 2011). Embora nesse experimento se utilizou os mesmos fungicidas para cada tratamento em todas as aplicações, é importante salientar sobre a necessidade de rotação de grupos químicos, ingredientes ativos, além da utilização de multissítios dentro dos programas de aplicação e sua importância.

Desta forma, o presente trabalho teve por objetivo avaliar a eficácia de fungicidas para o controle da Mancha-alvo na cultura da soja.

\section{MATERIAL E MÉTODOS}

Local: O experimento foi conduzido na estação experimental da Agro Carregal Pesquisa e Proteção de Plantas, no talhão 3, sob as coordenadas: S 1747'1,30" e W $51^{\circ} 0$ '8,10" e altitude de 753,4m.

Cultivar: Utilizou-se a cultivar CD 2728, a qual é suscetível a esse patógeno. Tal cultivar é de hábito de crescimento indeterminado, grupo de maturação 7.2, perfazendo 112 dias de ciclo na região. Foram mantidas 19 plantas por metro, totalizando 380 mil plantas por hectare com espaçamento de $50 \mathrm{~cm}$ entre linhas.

Data de semeadura: 20/10/2019 e emergência em 25/10/2019.

Aplicações: Durante a primeira aplicação dos tratamentos que receberam fungicidas, não havia sintomas de Mancha-alvo, portanto, a aplicação foi preventiva. Realizou-se então a inoculação artificial do patógeno em todos os tratamentos. Para a aplicação de fungicidas utilizou-se pulverizador costal pressurizado a $\mathrm{CO}_{2}$ com pressão constante e vazão de $150 \mathrm{~L} \mathrm{ha}^{-1}$.

Tabela 1 - Dados sobre as aplicações de fungicidas. Agro Carregal, Rio Verde-GO, 2020.

\begin{tabular}{|l|c|c|c|c|}
\hline & $1^{a}$ aplicação & $2^{a}$ aplicação & $3^{a}$ aplicação & $4^{a}$ aplicação \\
\hline Data & $09 / 12 / 2019$ & $23 / 12 / 2019$ & $06 / 01 / 2020$ & $20 / 01 / 2020$ \\
\hline Estádio fenológico & R1 & R4 & R5.3 & R5.5 \\
\hline Dias após emergência & 45 DAE & 59 DAE & 73 DAE & 87 DAE \\
\hline
\end{tabular}

Tratamentos: A lista de tratamentos com as respectivas doses encontra-se na tabela 2. 
Tabela 2 - Lista de tratamentos. Agro Carregal, Rio Verde-GO, 2020.

\begin{tabular}{|c|c|c|c|}
\hline $\begin{array}{c}\mathbf{N}^{\text {o }} \\
\text { tratamento }\end{array}$ & Produtos & Dose (mL ou g ha $\left.{ }^{-1}\right)$ & $\begin{array}{c}\text { Adjuvantes utilizados + doses } \\
(\mathrm{mL})\end{array}$ \\
\hline 1 & Testemunha & - & Áureo 375 \\
\hline 2 & $\begin{array}{c}\text { Bixafen + Protioconazol } \\
\text { Trifloxistrobina }\end{array}$ & $62,5+87,5+75$ & Assist 500 \\
\hline 3 & Fluxapiroxade + Protioconazol & $60+84$ & Strides 375 \\
\hline 4 & $\begin{array}{c}\text { Azoxistrobina + Tebuconazol }+ \\
\text { Mancozebe }\end{array}$ & $94+112+1194$ & Strides 375 \\
\hline 5 & $\begin{array}{c}\text { Azoxistrobina + Protioconazol }+ \\
\text { Mancozebe }\end{array}$ & $75+75+1050$ & Lanzar 450 \\
\hline 6 & Protioconazol + Fluindapyr & $84+84$ & \\
\hline
\end{tabular}

\section{RESULTADOS E DISCUSSÃO}

As condições ambientais foram favoráveis à incidência de Mancha-alvo durante todo o ciclo da cultura, porém com menor severidade devido à menor temperatura em comparação com anos anteriores. Esse tipo de condição climática já foi observado em outros estudos, justificando o monitoramento climático para comparação com estudos prévios e futuros (Teramoto et al., 2012; De Carvalho Ribeiro et al., 2016). As doenças diagnosticadas nesse experimento foram a Mancha-parda e a Mancha-alvo. A incidência inicial foi de Mancha-parda, a qual foi suprimida pela Manchaalvo durante a fase de enchimento de grãos. Os primeiros sintomas de Mancha-alvo ocorreram apenas a partir do início de enchimento de grãos.

Em relação à severidade da Mancha-alvo, verificou-se em todas as avaliações que os fungicidas foram efetivos no controle da doença (tabela 3). Entretanto, foi na última avaliação em R7 que maiores diferenças foram verificadas entre os tratamentos.

Provavelmente em decorrência do próprio desenvolvimento mais tardio da doença em condições climáticas mais amenas como observado por Basso et al., 2015 ao realizar testes envolvendo Mancha-alvo e Antracnose na cultura da soja. A menor severidade da doença foi verificada nos tratamentos 2, 5 e 6 (tabela 3).

Analisando-se a área abaixo da curva de progresso da doença (AACPD) e eficácia de controle verificou-se que todos os tratamentos diferiram da testemunha, mas os tratamentos 2 e 5 foram os únicos que apresentaram eficácia acima de $80 \%$ de controle (tabela 4). Para desfolha todos diferiram da testemunha sem diferir entre si (tabela 4). 
Tabela 3 - Severidade da Mancha-alvo em função do controle químico. Agro Carregal, Rio Verde-GO, 2020.

\begin{tabular}{|c|c|c|c|c|}
\hline $\begin{array}{c}\mathbf{N}^{\mathbf{0}} \\
\text { tratamento }\end{array}$ & Tratamentos & $\mathbf{R 5 . 2}$ & $\mathbf{R 5 . 5}$ \\
\hline 1 & Testemunha & $4,25 \mathrm{~b}$ & $21,75 \mathrm{~b}$ & $54,00 \mathrm{~d}$ \\
\hline 2 & $\begin{array}{c}\text { Bixafen + Protioconazol } \\
\text { + Trifloxistrobina }\end{array}$ & $0,13 \mathrm{a}$ & $4,75 \mathrm{a}$ & $5,75 \mathrm{a}$ \\
\hline 3 & $\begin{array}{c}\text { Fluxapiroxade } \\
\text { Protioconazol }\end{array}$ & $0,50 \mathrm{a}$ & $7,25 \mathrm{a}$ & $10,50 \mathrm{bc}$ \\
\hline 4 & $\begin{array}{c}\text { Azoxistrobina }+ \\
\text { Tebuconazol + } \\
\text { Mancozebe }\end{array}$ & $0,63 \mathrm{a}$ & $8,25 \mathrm{a}$ & $11,75 \mathrm{c}$ \\
\hline 5 & $\begin{array}{c}\text { Azoxistrobina }+ \\
\text { Protioconazol + } \\
\text { Mancozebe }\end{array}$ & $0,13 \mathrm{a}$ & $5,75 \mathrm{a}$ & $7,00 \mathrm{ab}$ \\
\hline 6 & $\begin{array}{c}\text { Protioconazol }+ \\
\text { Fluindapyr }\end{array}$ & $0,25 \mathrm{a}$ & $6,75 \mathrm{a}$ & $8,25 \mathrm{abc}$ \\
\hline
\end{tabular}

Médias seguidas pela mesma letra em cada coluna não diferem estatisticamente entre si pelo teste de Tukey a $5 \%$ de probabilidade.

Tabela 4 - Área baixo da curva de progresso da doença, eficácia (\%) e desfolha por Mancha-alvo em função do controle químico. Agro Carregal, Rio Verde-GO, 2020.

\begin{tabular}{|c|c|c|c|c|}
\hline $\mathbf{N}^{\circ}$ tratamento & Tratamentos & AACPD & Eficácia & Desfolha (\%) \\
\hline 1 & Testemunha & $674,38 \mathrm{~d}$ & - & $85,00 \mathrm{~b}$ \\
\hline 2 & $\begin{array}{c}\text { Bixafen + Protioconazol }+ \\
\text { Trifloxistrobina }\end{array}$ & $102,38 \mathrm{a}$ & 84,82 & $53,75 \mathrm{a}$ \\
\hline 3 & $\begin{array}{c}\text { Fluxapiroxade }+ \\
\text { Protioconazol }\end{array}$ & $169,63 \mathrm{bc}$ & 74,85 & $58,75 \mathrm{a}$ \\
\hline 5 & $\begin{array}{c}\text { Azoxistrobina }+ \\
\text { Tebuconazol + Mancozebe }\end{array}$ & $192,13 \mathrm{c}$ & 71,51 & $62,50 \mathrm{a}$ \\
\hline 5 & $\begin{array}{c}\text { Azoxistrobina + } \\
\text { Protioconazol + } \\
\text { Mancozebe }\end{array}$ & $124,00 \mathrm{ab}$ & 81,61 & $53,75 \mathrm{a}$ \\
\hline 6 & Protioconazol + Fluindapyr & $146,50 \mathrm{abc}$ & 78,28 & $61,25 \mathrm{a}$ \\
\hline & C.V. (\%) & 12,42 & - & 8,52 \\
\hline
\end{tabular}

Médias seguidas pela mesma letra em cada coluna não diferem estatisticamente entre si pelo teste de Tukey a 5\% de probabilidade.

Na figura 1 verifica-se diferenças de severidade entre os tratamentos principalmente em relação à desfolha. $\mathrm{Na}$ testemunha também podem ser verificados os sintomas das doenças nas folhas do terço superior e intensa desfolha. Apesar do bom controle, os tratamentos 2 e 6 apresentaram adiantamento no ciclo da cultura.

Considerando-se os parâmetros de rendimento, verificou-se que todos os tratamentos diferiram da testemunha em relação ao peso de mil grãos, sem diferir entre si (tabela 5). Segundo Nomelini et al. (2010), a massa de 1000 grãos é um dado que pode ser utilizado na comparação da qualidade dos grãos, apesar de ser comumente utilizada em ensaios, como componente de rendimento de diversos cultivos, pode ser útil como fator para comparação de qualidade de sementes. Quanto à produtividade apenas os tratamentos 5 e 6 diferiram da testemunha, embora não tenham diferido dos demais tratamentos contendo fungicidas. Como houve forte correlação negativa entre severidade e produtividade $(-94,52 \%)$, deve se considerar o incremento relativo proporcionado pelo 
uso de fungicidas. Todos os tratamentos apresentaram incrementos superiores a 5,72 sc ha-1 (tratamento 3),

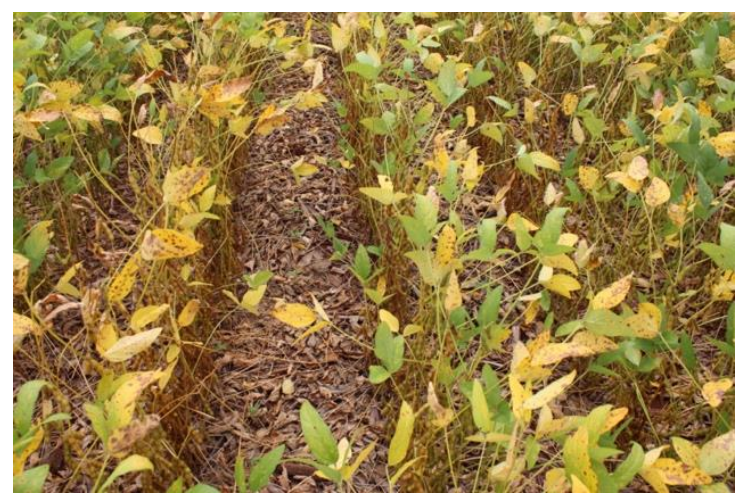

Testemunha

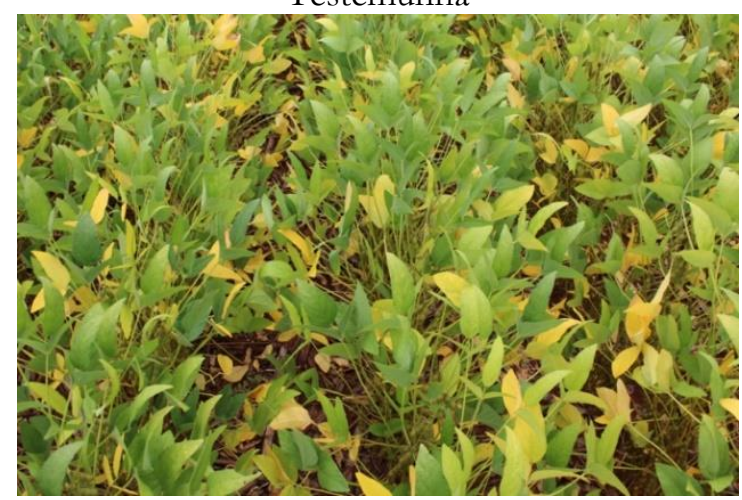

Fluxapiroxade + Protioconazol

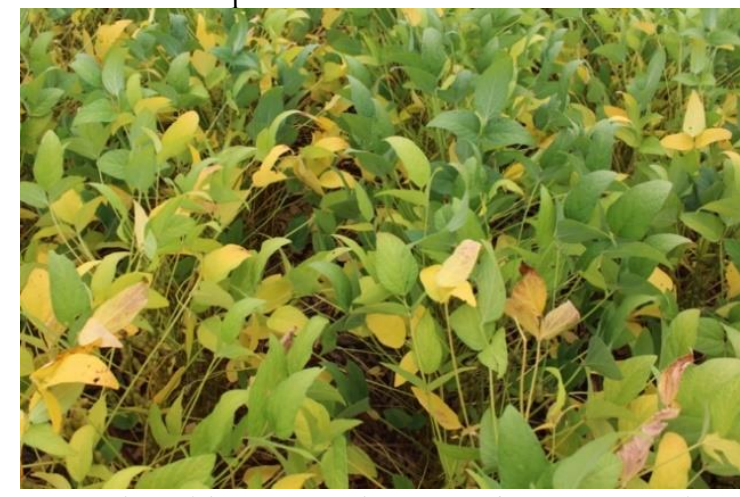

Azoxistrobina + Protioconazol + Mancozebe sendo que o tratamento 5 destacou-se com incremento de 9,79 sc ha-1 $^{-1}$ (tabela 5).

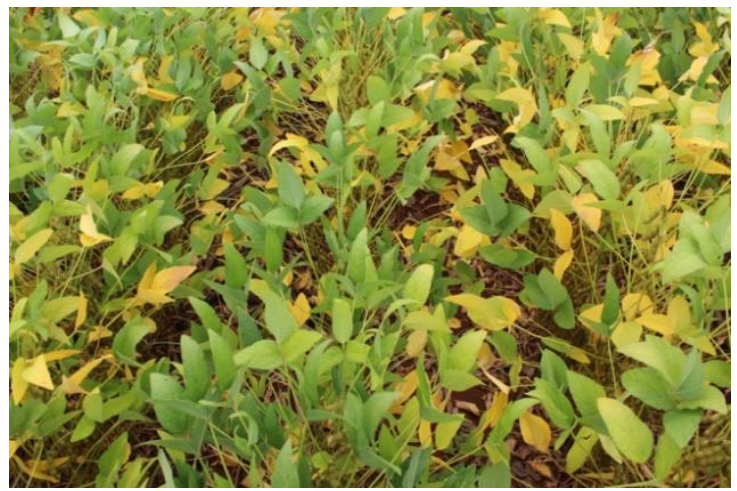

Bixafen + Protioconazol + Trifloxistrobina
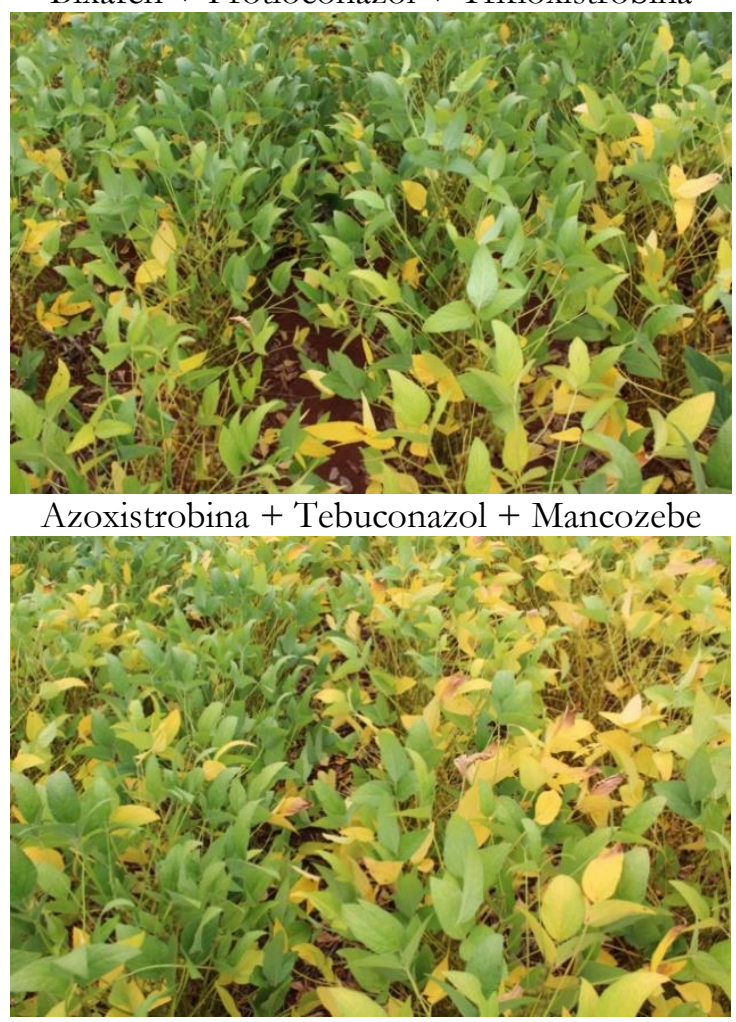

Protioconazol + Fluindapyr

Figura 1 - Fotos das parcelas. Agro Carregal, Rio Verde-GO, 2020.

Tabela 5 - Peso de mil grãos, produtividade e incremento produtivo em função do controle químico da Manchaalvo. Agro Carregal, Rio Verde-GO, 2020.

\begin{tabular}{|c|c|c|c|c|}
\hline $\mathbf{N}^{0}$ tratamento & Tratamentos & PMG (g) & Produtividade $\left(\mathrm{sc} \mathrm{ha} \mathrm{a}^{-1}\right)$ & Incremento (sc ha-1) \\
\hline 1 & Testemunha & $138,39 \mathrm{~b}$ & $69,70 \mathrm{~b}$ & - \\
\hline 2 & $\begin{array}{c}\text { Bixafen }+ \\
\text { Protioconazol }+ \\
\text { Trifloxistrobina }\end{array}$ & 147,23 a & $78,00 \mathrm{ab}$ & 8,31 \\
\hline 3 & $\begin{array}{c}\text { Fluxapiroxade }+ \\
\text { Protioconazol }\end{array}$ & $145,66 \mathrm{a}$ & $75,42 \mathrm{ab}$ & 5,72 \\
\hline 4 & $\begin{array}{c}\text { Azoxistrobina }+ \\
\text { Tebuconazol }+ \\
\text { Mancozebe }\end{array}$ & 150,06 a & $77,55 \mathrm{ab}$ & 7,86 \\
\hline
\end{tabular}




\begin{tabular}{|c|c|c|c|c|}
\hline 5 & $\begin{array}{c}\text { Azoxistrobina }+ \\
\text { Protioconazol }+ \\
\text { Mancozebe }\end{array}$ & 150,28 a & 79,49 a & 9,79 \\
\hline 6 & $\begin{array}{c}\text { Protioconazol }+ \\
\text { Fluindapyr }\end{array}$ & $147,60 \mathrm{a}$ & $78,56 \mathrm{a}$ & 8,87 \\
\hline C.V. $(\%)$ & 1,42 & 5,03 & - \\
\hline
\end{tabular}

Médias seguidas pela mesma letra em cada coluna não diferem estatisticamente entre si pelo teste de Tukey a 5\% de probabilidade.

\section{CONCLUSÕES}

A Mancha-alvo tem se tornado cada vez mais importante no sudoeste goiano, embora não seja a principal doença na região. A não utilização de fungicidas para o manejo dessa doença pode acarretar perdas de até $20 \%$.

Nessa safra especificamente as perdas foram menores, provavelmente pelas condições de temperatura que foram inferiores a anos anteriores. Houve aumento significativo da severidade na fase final de enchimento de grãos. Foi observada também alta eficácia nos tratamentos 5 e 6 , porém percebeu-se um adiantamento no ciclo da cultura nos tratamentos 2 e 6 .

\section{REFERÊNCIAS BIBLIOGRÁFICAS}

Amorim, L.; Rezende, J.A.M.; Bergamin Filho, A. (Eds.). Manual de Fitopatologia: princípios e conceitos. v.1. São Paulo: Editora Agronômica Ceres. 2011. 704p

Avozani, Aveline; Reis, Erlei Melo; Tonin, Rosane Baldiga. Sensitivity loss by Corynespora cassiicola, isolated from soybean, to the fungicide carbendazim. Summa Phytopathologica, v. 40, n. 3, p. 273-276, 2014.

De Carvalho Ribeiro, Francisco et al. Associação de fungicida protetor com fungicidas sistêmicos no controle de mancha-alvo na cultura da soja. Revista verde de agroecologia e desenvolvimento sustentável, v. 11, n. 5, p. 5156, 2016.

Flor, Marcus Vinicius Cunha et al. Controle químico da mancha alvo (Corynespora Casiicola) na cultura da soja, safra 2017/18. In: IV SICTEGSemana Integrada de Ciência e Tecnologia de Gurupi. 2018.
Godoy, C. V. et al. Eficiência de fungicidas para o controle da mancha-alvo, Corynespora cassiicola, na safra 2006/07: resultados sumarizados dos ensaios cooperativos. Embrapa Soja-Circular Técnica (INFOTECA-E), 2007.

Godoy, C. V. et al. Eficiência de fungicidas para o controle da mancha-alvo, Corynespora cassiicola, na cultura da soja, na safra 2019/2020: resultados sumarizados dos ensaios cooperativos. Embrapa Soja-Circular Técnica (INFOTECA-E), 2020.

Marques, Guilherme; Terniski, Wallison José. Avaliação da eficiência de fungicidas sistêmicos associados a multissítios após simulação de chuva no controle de mancha-alvo (Corynespora cassiicola) na cultura da soja (Glicine max L. Merrill). 2019.

Nomelini, Q.S.S.; Costa, D.A.; Silva, L.F.; Ferreira, A.S.; Pereira, J.M.; Biase, N.G. Validação do método de dimensionamento do número médio ideal de sementes por saca. 2010. Disponível em: Acesso: $30 / 01 / 2014$

Sentelhas, P.C., Battisti, R., Câmara, G.M.S., Farias, J.R.B., Hampf, A., Nendel, C., 2015.The soybean yield gap in Brazil - magnitude, causes and possible solutions for a sustainable production. Journal of Agricultural Science 153, 1394-1411. https://doi.org/10.1017/S0021859615000313.

SOARES, Rafael M.; GODOY, Cláudia V.; OLIVEIRA, Maria Cristina N. de. Escala diagramática para avaliação da severidade da mancha alvo da soja. Tropical Plant Pathology, v. 34, n. 5, p. 333-338, 2009.

St-Marseille, A-F.G., Bourgeois, G., Brodeur, J., Mimee, B., 2019. Simulating the impacts of climate change on soybean cyst nemoted and dristibution of soybean. Agricultural and Forest Meteorology, v. 264, p. 178-187. 
https://doi.org/10.1016/j.agrformet.2018.1 0.008 .

TERAMOTO, A. et al. Sensibilidade a fungicidas de isolados de corynespora cassiicola provenientes do Estado de Goiás. In: Embrapa Soja-Artigo em anais de congresso (ALICE). In: CONGRESSO BRASILEIRO DE SOJA, 6., 2012, Cuiabá. Soja: integração nacional e desenvolvimento sustentável: anais. Brasília, DF: Embrapa, 2012., 2012.

TERAMOTO, Adriana et al. Sensibilidade de Corynespora cassiicola isolado de soja a fungicidas in vitro e controle químico de mancha-alvo da soja no campo. Summa Phytopathologica, v. 43, n. 4, p. 281-289, 2017. 